\title{
Research on WebGIS-Based Stadium Intelligent Service System
}

\author{
Guofeng-WU and Zhishuang-Li \\ Shijiazhuang Tiedao University, Shijiazhuang, Hebei, China, 050081 \\ ${ }^{[1]}$ wuguofeng@163.com
}

\begin{abstract}
Centered on the WebGIS technology of J2EE and combined with the distributed GIS special database, this paper researches on the WebGIS-based stadium intelligent service system. Through deep research of multiple traditional optimal path algorithms, improved algorithm is worked out. Then, after in-depth analyses of users' travel modes, this paper finally devises the optimal path of accessibility of stadiums which can be realized in the WebGIS-based stadium service system. The results show that this optimal path design makes it easier for users to check real-time stadiums and helps users achieve the most accurate information about stadiums, hotels, which provides convenience for users to make the best decisions and improves the efficiency of urban transport. Taking full advantage of mobile Internet and GPS technology, this design lays a foundation for the construction of smart city.
\end{abstract}

Keywords: WebGIS; stadiums; optimal path design

\section{Introduction}

Physical Education is a symbol of social development and progress of human civilization, the level of Physical Education is an important manifestation of the comprehensive national strength and the degree of social civilization. GIS-based city stadium system, as part of the smart city thematic applications, focuses on the accessibility analysis of spatial objects. With the advancement of modern technologies in Physical Education (modern technologies in Physical Education refers to a variety of related technologies currently existing in the field of sports which includes modern educational technology and geographical information technology), stadiums and geographic information technologies have more closely integrated with each other and researches in this area are also at the stage of progressive development.

In the research field of foreign countries, scholars embedded web technology into the studies of city stadiums and combined with the studies of digital maps. Such studies had made some achievements. However, neither the combined technology of GPS and electronic map had been embedded, nor the "4A" service concept of GIS had been applied to the city stadium. Toronto sports venues in Canada and the University of Michigan kendo in America have adopted the expression methods of modern digital maps. These methods mainly refer to geographic information methods based on vector expressing elements, which put such information as address, place names and other supporting information including practice areas and parking place etc. into digital maps ${ }^{[1]}$. Geographic expression method which is a combination of regional maps and web technologies is applied to stadiums in schools of Okayama Prefecture, Japan. Designers partition Okayama Prefecture based on geographic area. Areas with a stadium there are coated with a color, and then the map is embedded in a web page. There are descriptions about the names of stadiums presented on the map and links to detailed information webpage of each stadium ${ }^{[2]}$. The special thematic GIS about ski made by European ski resort, Khao Erxi $\mathrm{Na}$ of Dorothy Hill in Italy, established a thematic GIS website Including multiple cultural information and geographical information. In addition to a 
large number of text messages, this website also carefully designed geographic information databases of summer and winter under the guidance of the thought of geographic information visualization ${ }^{[3]}$.

At present, researches on city stadiums at home have integrated the geographic information technology with urban stadiums, which is mainly applied to the areas of the best site selection, spatial accessibility, site planning and system design and implementation and so on. Ma Yunchao and Sun Jinhai developed an embedded geographic information system (GIS) and a city sports facilities information system of panoramic technology ${ }^{[4]}$. Peng Lixin, Guo Weiyong embedded the road smooth coefficient into their study and established the best location model of stadium based on average shortest path ${ }^{[5]}$. Cheng Shijun, in "Modern Physical Education Art", mainly analyzed the instances of using GIS technology for geographic expression in current sports areas. By using geographic information expression method combing regional map with web technology, geographic information expression method combing digital intelligent maps with page production technology and thematic GIS website based on network including multiple cultural information and geographic information, he pointed that individual users of GPS products now apply GPS equipment to sports tourism, cross-country, field excursions, long-distance travel by car and climbing ${ }^{[6]}$. Chen Peng, Wang Xiaoxuan, Liu Miaolong first designed a stadium-based crowd evacuation simulation model, worked out the shortest path algorithm with smart audience as the main subject and developed an analog system based on multi-agent system and GIS integration. Finally they took Shanghai Stadium as an example and run a dynamic simulation on the crowd departure process ${ }^{[7]}$.

Based on the previous researches, this paper employed new technologies of WebGIS and mobile GIS, which will involve the study and implementation of such key technologies as real-time navigation and positioning, data storage, buffer analysis algorithm, the optimal path design algorithm. There are two differences between this system and the traditional intelligent guide system. On the one hand, this system is a realization mode based on B/S webpage and does not need load plug-in; on the other hand, this paper focuses on the study and implementation of spatial analysis algorithm for path planning, which is not shown in the intelligent guide system.

\section{General Structure Design of the System}

This system takes the users' needs as starting point and highlights the features of GIS in stadium service. On the one hand, making functions cater for the flexible application of users and meeting the maximum needs of users for stadiums; on the other hand, centered on GIS, making the best use of network information technology and spatial database technology ${ }^{[8]}$.

This system adopts MVC (Mode-View-Controller) design mode of J2EE and achieves system implementation through Hibernate + JSP + Servlet technology system. The foreground of the system is implemented by using HTML5 + Javascript + CSS. As shown in Figure 1, the system is designed and implemented by adopting the four-story mode of $\mathrm{B} / \mathrm{S}$. The developing and operating environment of the system is as follows:

Development environment: JDK1.6, EditPlus, Eclipse IDE for Java EE Developers

Operating system: Win7 Ultimate

Database: Oraclellg, Geodatabase

Development languages and platforms: Java, JavaScript

Web Application Server: Tomcat 6.0

Server GIS: ArcGIS Server 10.0 


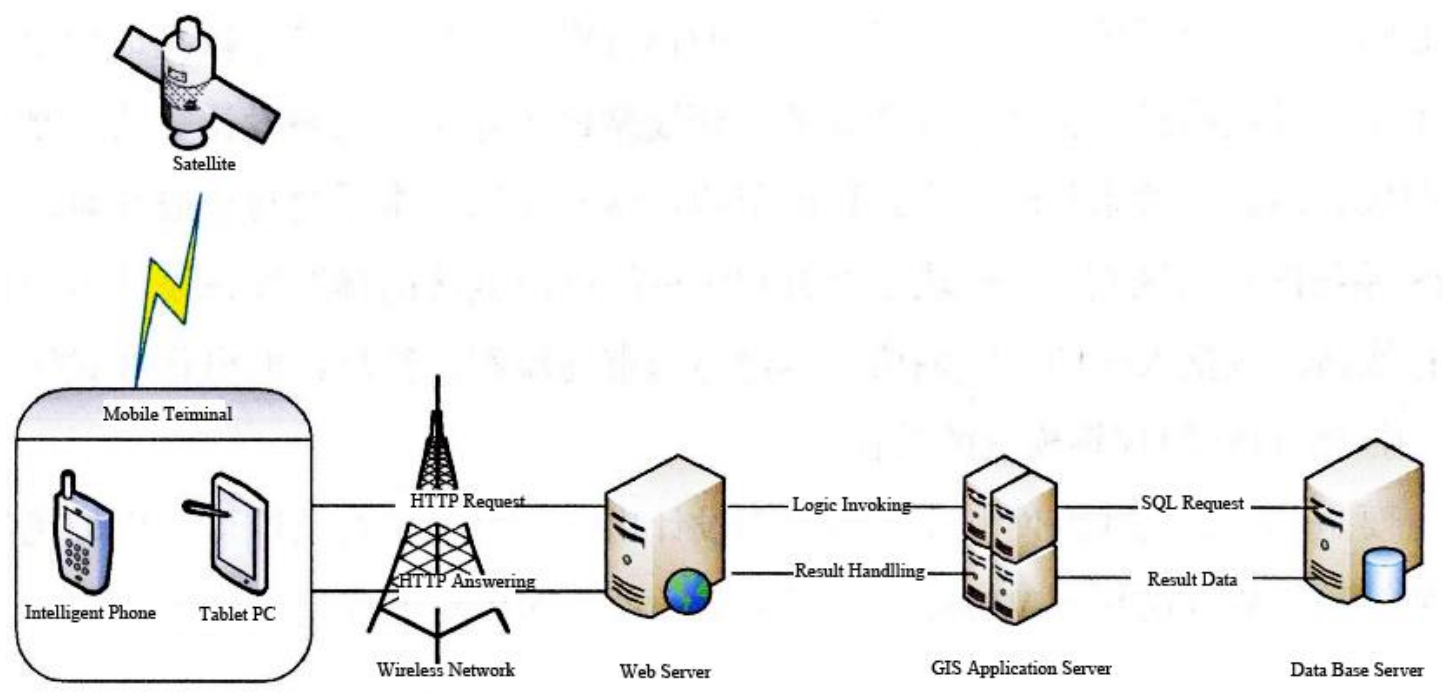

Figure 1. System Structure Diagram

(1) client-side

Smart mobile terminals in a way of wireless communication access to computer through wireless gateway. The mobile terminals get the longitude and latitude, time and displacement speed of the current location through analyzing the received GPS data ${ }^{[9]}$. It transmits the data to the back-end Web server which invokes GIS server to make positioning calculation. Then the raster image that centers on that position is formed and the image is transmitted to the mobile terminals, which finally makes the location service available.

(2) Web Server

Web server mainly provides venue for the operating of server software, and at the same time it also uses GIS server and database server for data communication. Users store the data package to be requested in the format of XML. Web server deploys a Servlet to serve as the controller, and then it adopts DOM parsing technology to analyze the requested data package and gets request packet command words. Based on the value of the command words, it determines to execute corresponding business operations and thereby distributes the requests to GIS server and database server. As the container of the Servlet and JSP files, Web sever is primarily responsible for the interaction between client-sides and each server.

(3) GIS application server

GIS application server provides site for the operation of GIS server software. On the one hand, it responses to the data processing requests from Web server; on the other hand, it can perform query and spatial analysis operation on geographic data. It can also process requests from foreground through adopting Geoprocessing services released by ArcGIS Server.

(4) The database server

The database server receives SQL data requests sent from GIS application server and then returns the results data obtained by data processing to GIS application server.

\section{Database Design and Construction}

\subsection{GIS Spatial Database Architecture}

GIS database is a kind of database that expresses, stores and manages certain spatial information obtained from geographical space through specific information structure (such as land, planning, environment, transportation, etc.) and data model (such as the 
relational model, object-oriented model, etc.) so as to meet the demands of different Internet / intranet users for spatial information ${ }^{[10]}$.

According to the theme, it intercepts information at different spatial and temporal scales from different GIS application system. The information ranges from the transient to the zone and to the overall geographic information systems ${ }^{[11]}$. Distributed database is the use of computer networks to connect databases which are physically dispersed but which need different degrees of concentration in managing and controlling, which finally forms a unified spatial data management system, as shown in Figure 2.

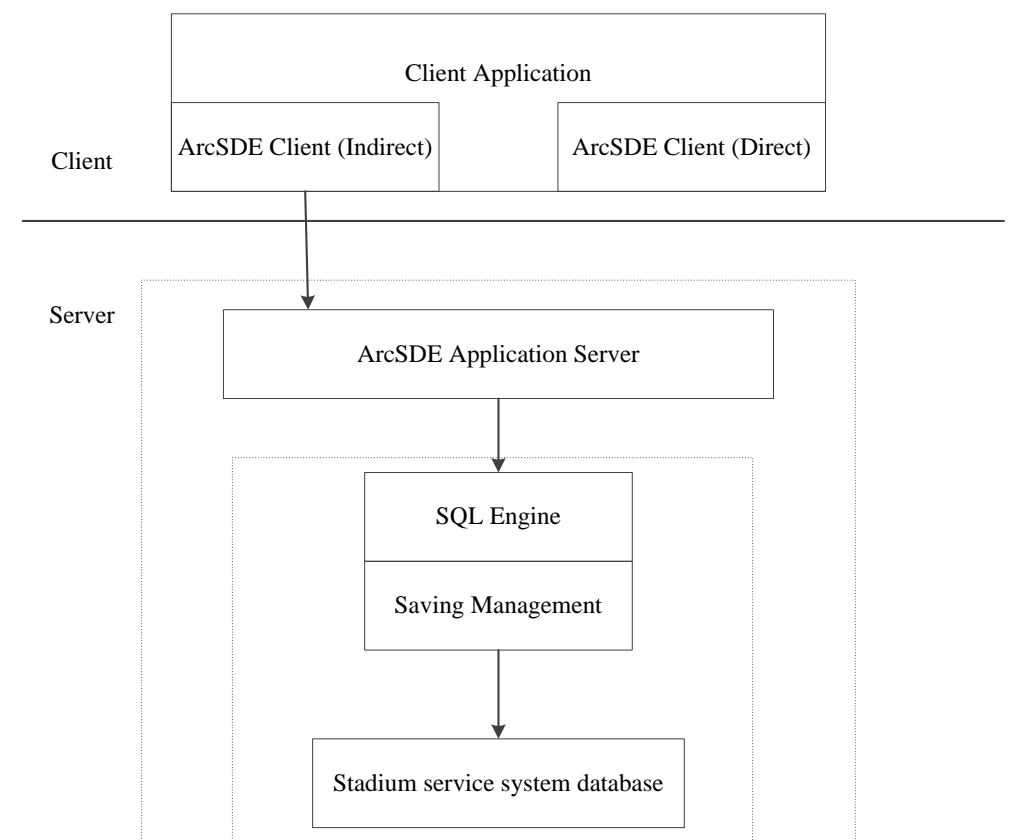

Figure 2. Data Access based on ArcSDE

\subsection{Spatial Database}

After getting familiar with the architecture and installing the software, the processed spatial data can be imported to Geodatabase which is the backend database of the system through spatial database engine ArcSDE, specific steps include:

(1) Creating a database instance. The database instance in this article is Shijiazhuang stadiums database;

(2) Building three new geographic feature datasets which can be divided into: transportation thematic datasets, basic geographic datasets and stadiums thematic datasets. Then setting the spatial reference coordinate system as WGS-84 coordinate system;

(3) Importing the data.

\subsection{Distributed Realization of System Database}

To sum up, architectures of distributed spatial database are as follows:

(1) Extending the spatial data accessibility on the basis of traditional spatial database and making it cater for the needs of distributed systems;

(2) Since spatial metadata is considered as a key technology of spatial information sharing, the architecture of distributed database can be implemented by using metadata technology;

(3) Using unified spatial data model and unified spatial data standards to implement the 
architecture of distributed spatial data.

In the experiments of this paper, Shijiazhuang City Stadium is taken as the object of the research. Since data comes from a variety of different ways, the data of stadium is implemented by distributed ways, just as shown in Figure 3.

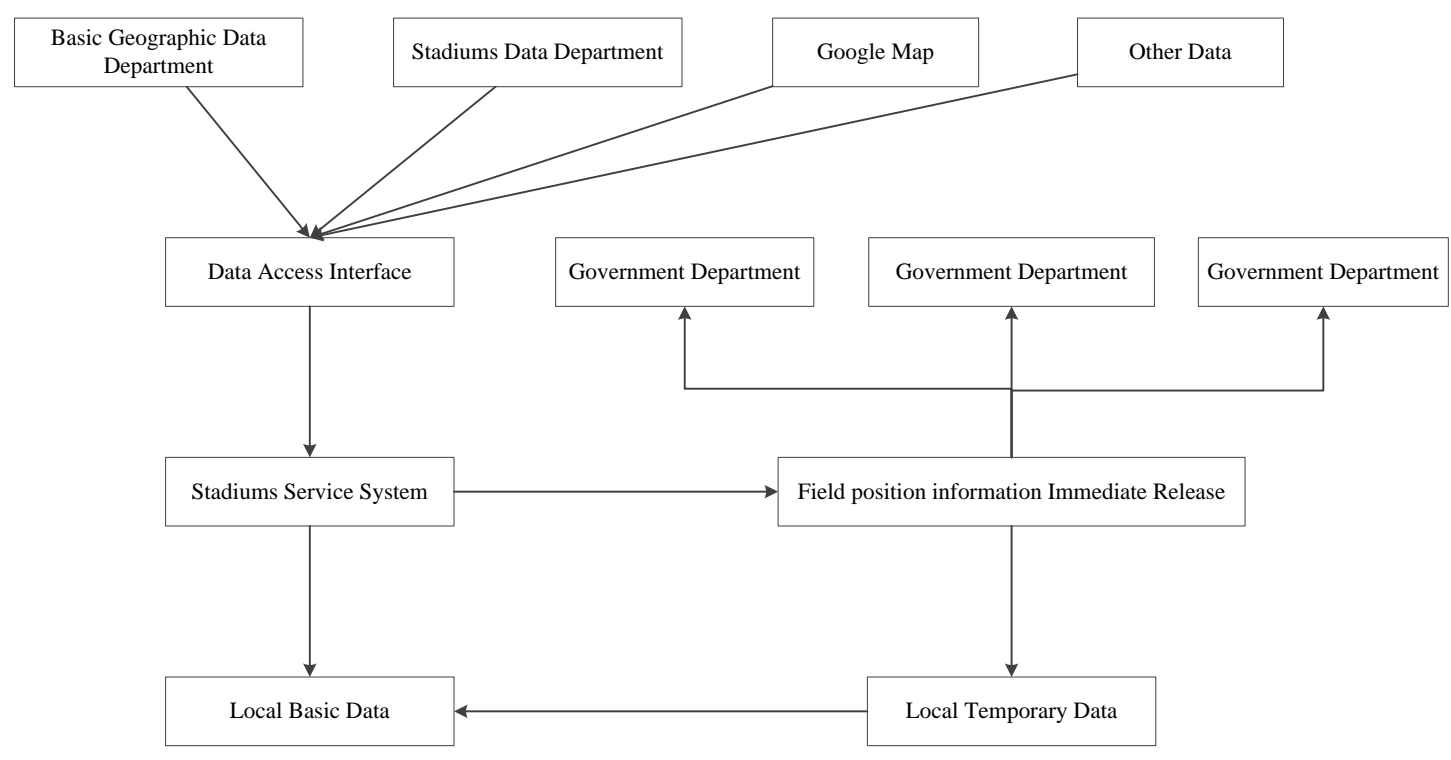

Figure 3. Distributed Database Structure Diagram in Shijiazhuang Stadium

In order to obtain data from outside, various data access interfaces are preset. Relevant data from different data departments distributed in different sites can be obtained through these interfaces; local data can be stored in multiple data servers. Each data server manages the data separately and then transfers the data management functions from the system platform to each sever, which enables the system itself to focus on the stadium-based spatial analysis and field information distribution; finally, the real-time field position information can be distributed to stadiums administrators, users, and the relevant responsible personnel through the system platform.

\section{Design of the Optimal Travel Route Algorithm}

\subsection{Traditional Shortest Path Algorithm}

Commonly used shortest path algorithms include: Dijkstra algorithm and Bellman-Ford algorithm. Wherein, Dijkstra algorithm is also known as single-source shortest path ${ }^{[12]}$.

The basic idea of Dijkstra algorithm is supposed that each point has a corresponding marker ${ }^{[13]}$. Where the length from the source point $s$ to the target point $\mathrm{j}$ is the shortest path; $\mathrm{Pj}$ is the point prior to point $\mathrm{j}$ which is one of the points of the shortest path from $\mathrm{s}$ to $\mathrm{j}$. The solving process of shortest path algorithm from the source point $s$ to the target point $\mathrm{j}$ is as shown in Figure 4. 


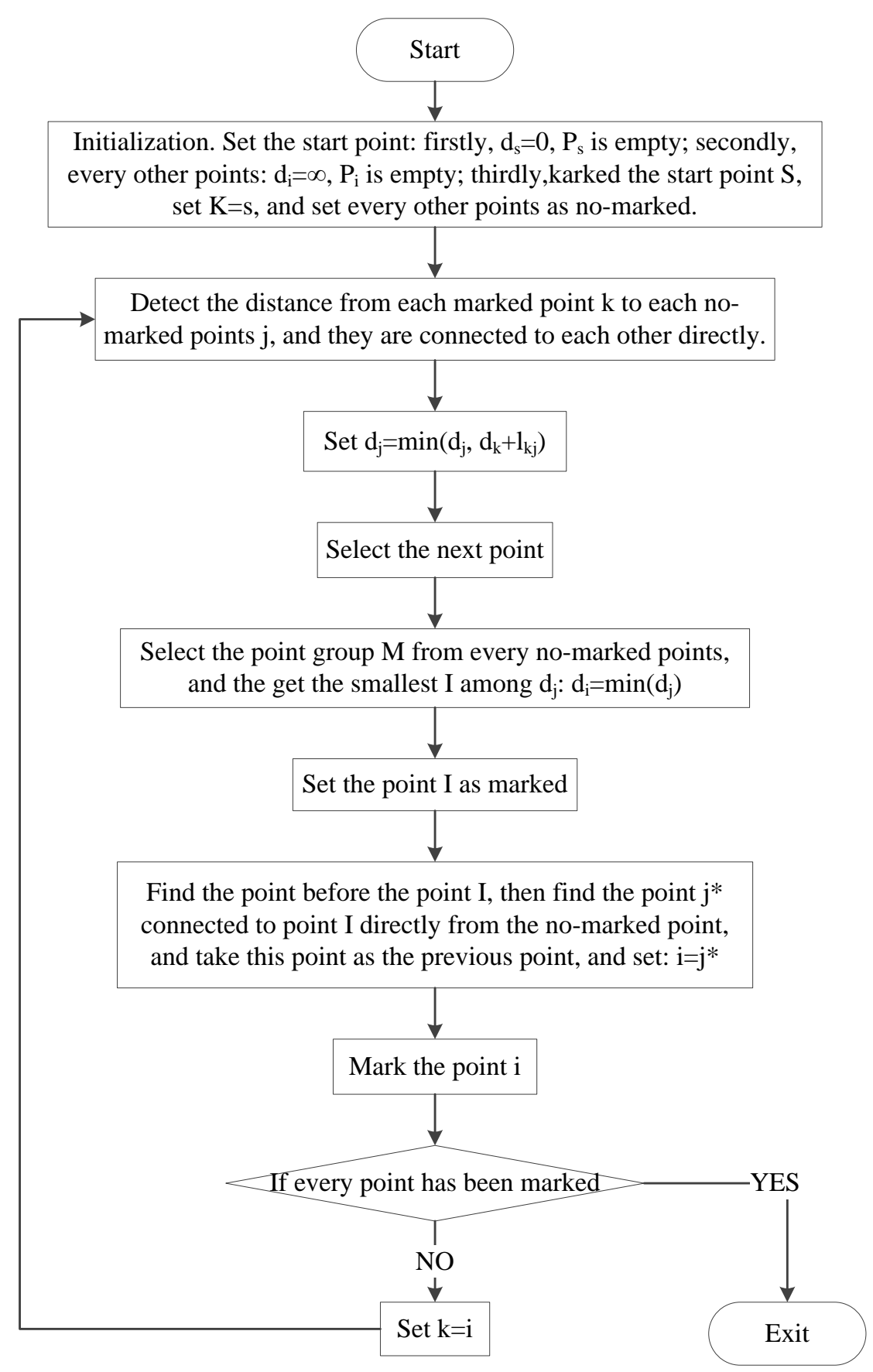

Figure 4. Shortest Path Algorithm based on Point Mark

\subsection{Improved Shortest Path Algorithm}

Seen from this algorithm process, the core of it is to find a minimum arc between the source point and the final point through constantly marking the nodes. However, there is a problem here.To achieve this algorithm, all nodes in the traffic network should be scanned. In the case involved mass data, the more nodes are, the more numbers of cyclic operations are, which will seriously affect the computation time of the algorithm.

In this case, considering the two factors of time and space, we found a solution idea to solve this problem. The effective approach is as follows:

(1) Ranking the nodes to be scanned and marked in order of the size of the arcs in which they located. In this way, each cycle can only get the qualified nodes and irrelevant 
nodes will be omitted, which improves the execution efficiency of the algorithm.

(2) Adopting the rectangular area incremental approach to determine the scope of research.

Determining the scope of research through the rectangular area incremental approach and selecting traffic road network nodes in the range of the source point and final point, thereby avoiding computing all the nodes. When using rectangular area increment method to retrieve the path, some nodes which are not related to the shortest path searched currently can be filtered out. To determine whether a point is within the region, only the size of two numbers has to be compared. This method reduces the computation time on the one hand, and lowers the size and complexity of the algorithm on the other hand.

The specific implementation process is as follows: First, a user inputs source point A and destination point $\mathrm{B}$; second, the source point $\mathrm{A}$ and destination point $\mathrm{B}$ in the length of $\mathrm{P}=\mathrm{d}$ extend outwardly to $\mathrm{C}$ and $\mathrm{D}$; then searching the shortest path in the rectangular area $\mathrm{ABCD}$ by using Dijkstra algorithm; if the shortest path is obtained, and then displays it. If not obtained then determines whether $\mathrm{P}$ is out of range. If it does, the circulation should be exited. If not, $\mathrm{C}$ and $\mathrm{D}$ will be extended in the length of $\mathrm{P}=\mathrm{d}$ to another new $\mathrm{C}$ and D; back to the third step and then continue the circle. As shown in Figure 5.

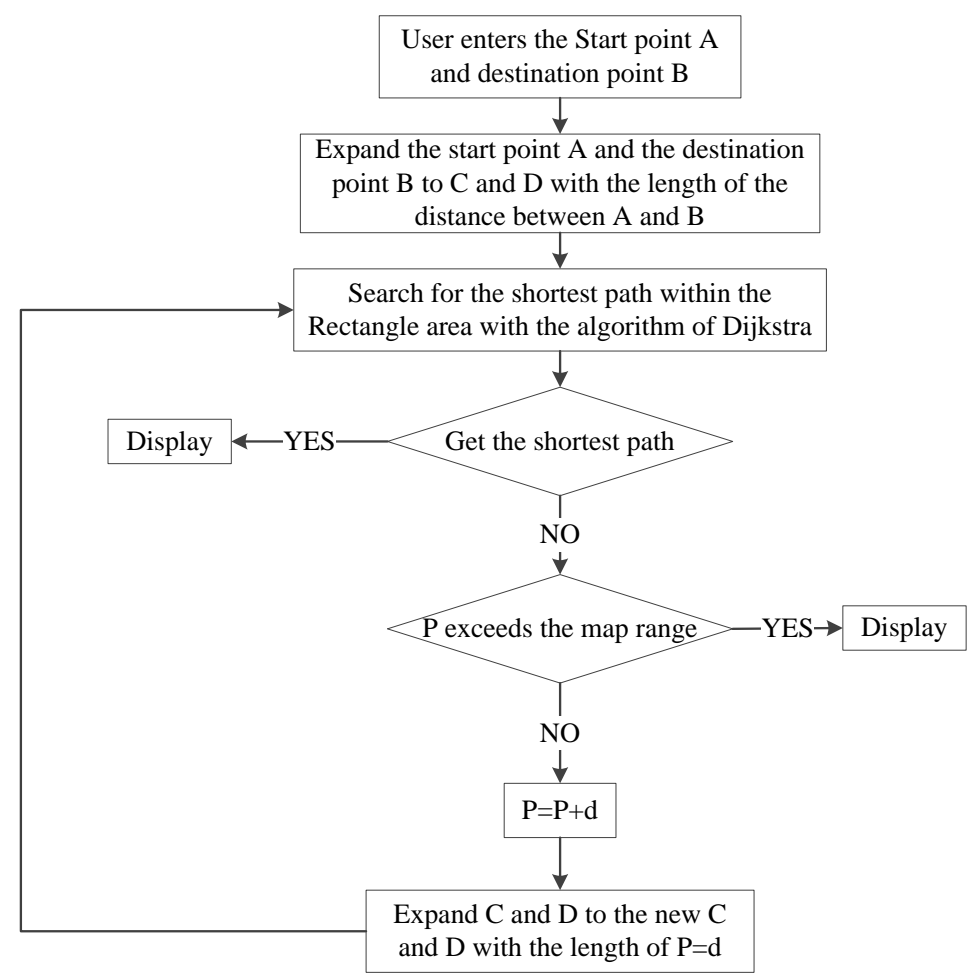

Figure 5. Shortest Path Search Flowchart based on Rectangular Area Incremental Method

\subsection{Realization of Optimal Travel Route Design Algorithm based on Stadium}

When large-scale events are held in Shijiazhuang City, users need to go to more than one stadium according to their own interests. The order of going to these stadiums is the users' independent choice, which involves the solving of the single-source multi-nodes problem. City-wide single-source multi-nodes distribution problem refers to the goods distribution problem of short-distance transports in the same city which is from a single supply point to two or more demand points. In order to ensure the lowest spatial operating costs and the highest travel time efficiency, an optimal stadium-based travel path should be designed. Since most users go out by bus, this algorithm is mainly based on bus lines. 
When involves many times of transfer, the algorithm will automatically converts to the shortest path algorithm which is based on marked nodes.

The optimal travel path design algorithm is the core of the system, which is also the biggest difference with the traditional intelligent navigation and guide service GIS system. This algorithm needs to work out a travel route according to the events in which the travelers participate. The travel line also includes the hotel to be booked and the trip mode to the hotel, etc. The algorithm invokes public transport transfer algorithm and takes the space constraints and time constraints of the travel route into consideration. Factors being considered in the algorithm include: single-source multi-node, optimal accessibility index.

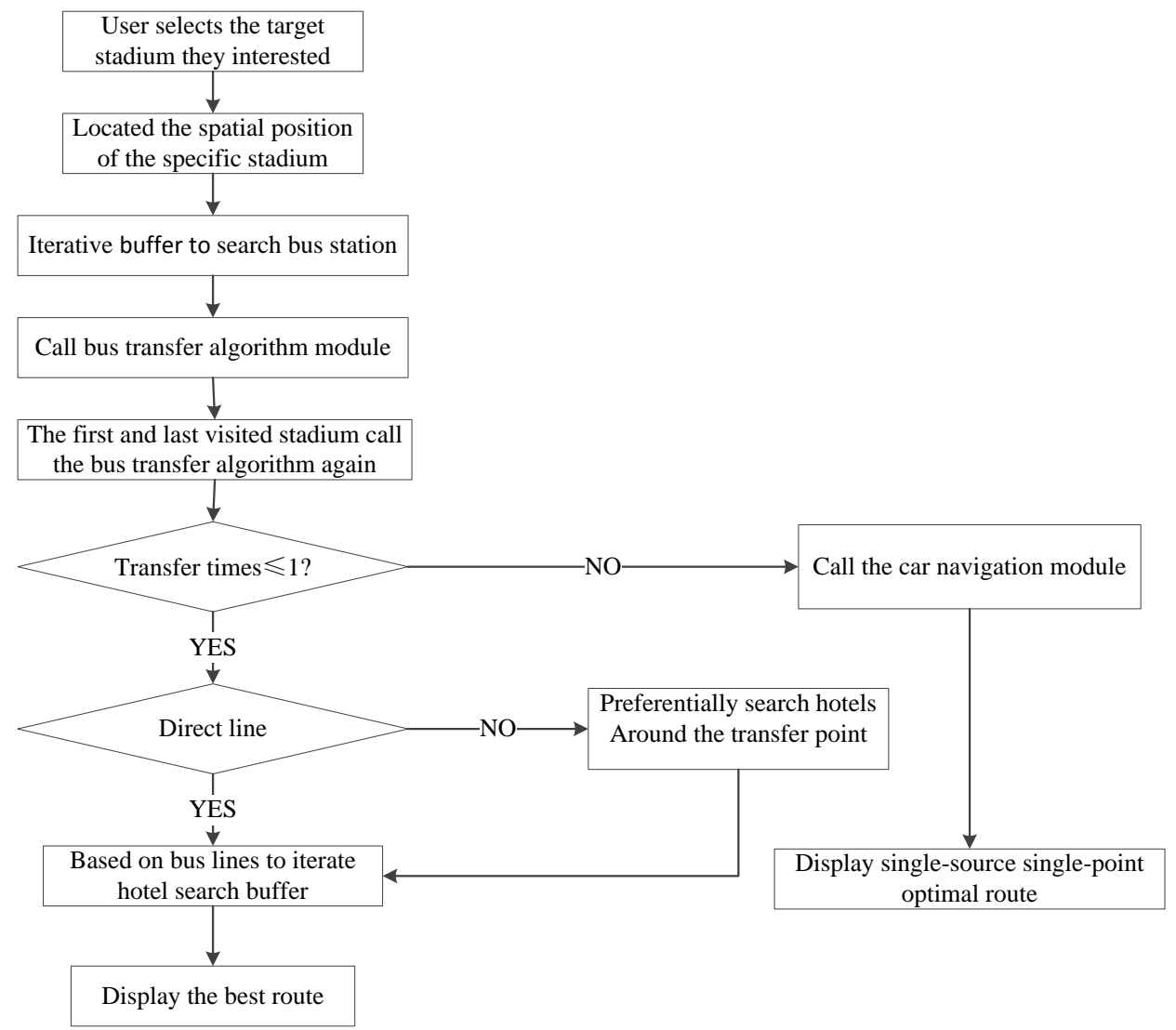

Figure 6. The Basic Idea of Circuit Design Algorithm

As shown in Figure 6, after users choose the item they are interested in, the system gets the spatial location of the stadiums requested by users through keyword retrieval technology. After getting the spatial location, the system looks for the bus station around the stadium in the fattest speed by adopting an iterative search buffer strategy. The so-called buffer iterative search refers to the fact that the buffer search range around the stadium is successively increased until it achieves success or it reaches the upper limit of the search range. This spatial search strategy not only speeds up the search space, but also to some extent controls the number of bus stations, which reduces the pressure of transfer.

\section{Design of WebGIS-based Mobile Intelligent Service}

\subsection{Realization of System Login and Registration Functions}

Login identities are divided into administrators and ordinary users. Ordinary users can only access to the functions of basic information query and stadiums path query. Except the privileges shared by ordinary users, administrators also enjoy the privilege of 
releasing field position information. This function is implemented by adopting JSP + JavaBean + Servlet three-tier architecture technology, and its technical route is as shown in Figure 7.

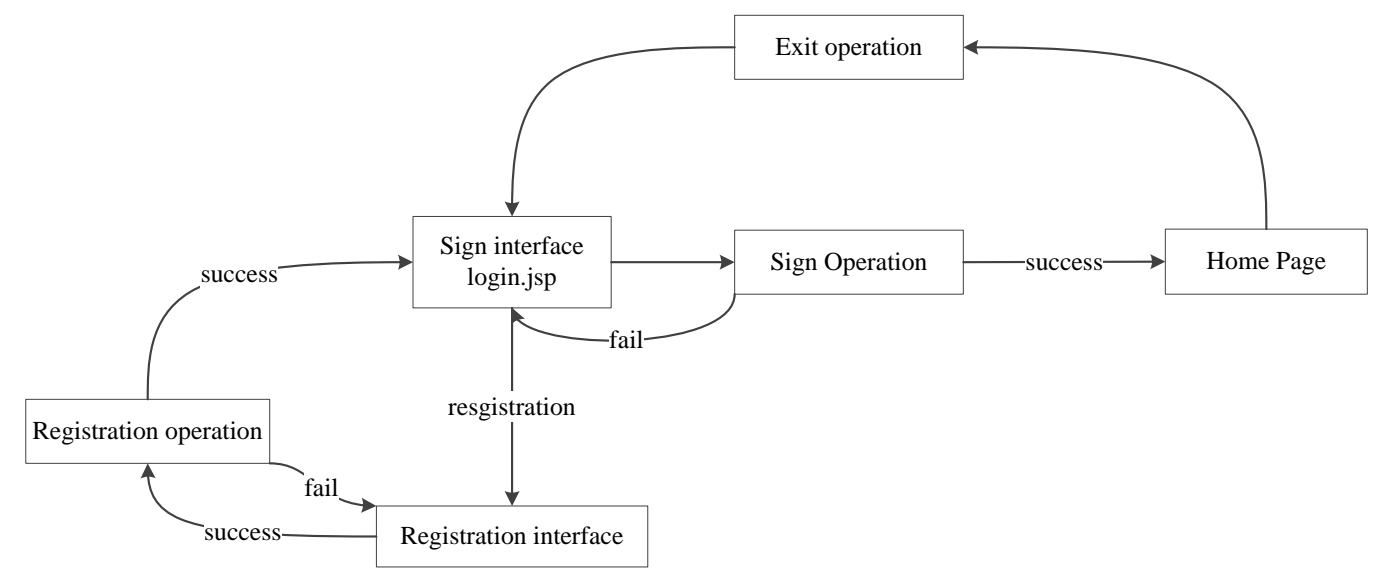

Figure 7. The Basic Operation Principle of Login Function

The login and register module adopts a combined approach of dynamic pages and static pages, its implementation process is as shown in Figure 8:

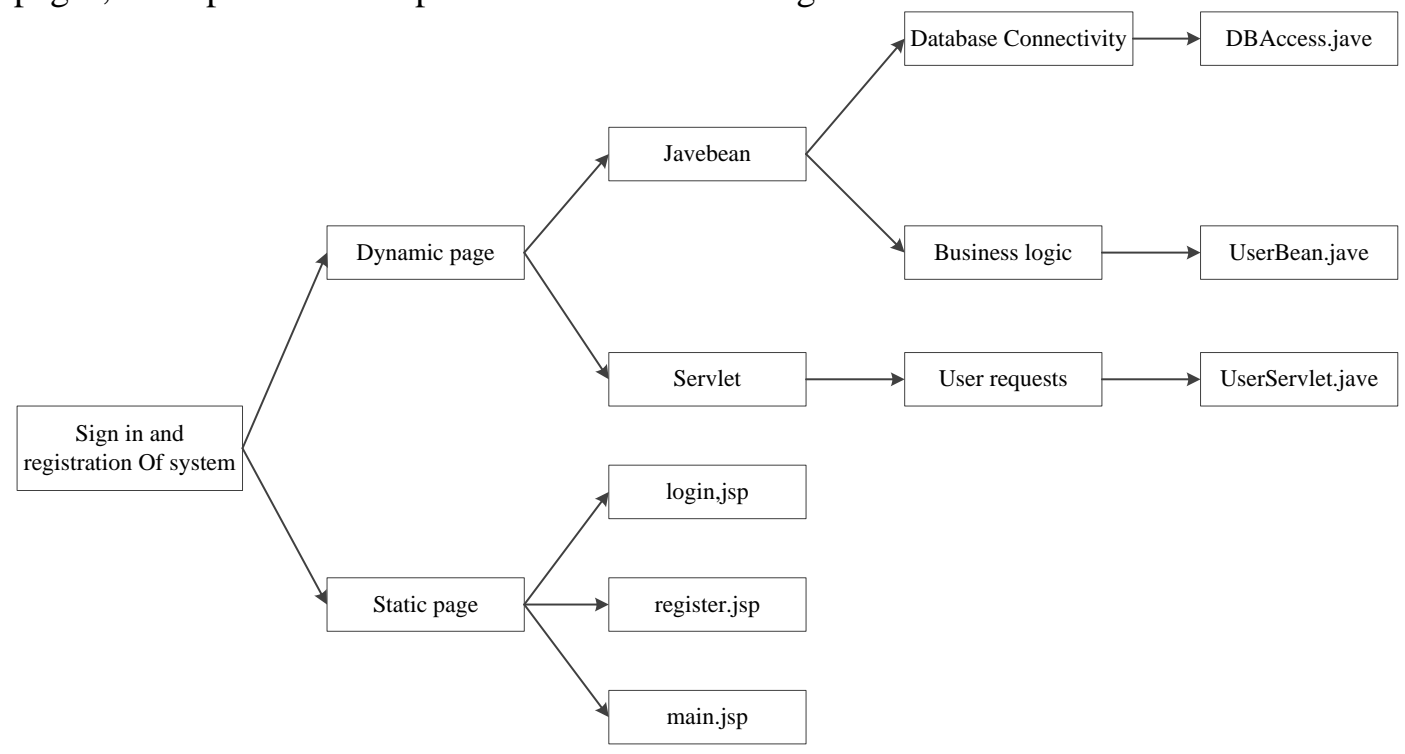

Figure 8. The Basic Documents Block Diagram of Registration Function

\subsection{The Invoking and Display of Map Service}

To invoke the map service of GoogleMap, the platform plug of GoogleMap API should be installed in Eclipse environment.

(1) Get MD5 fingerprint according to debug. Keystore

keytool-list-alias androiddebugkey

-keystore "C: \Users \ Administrator \ .android \debug.keystore" -storepass android -keypass android

(2) Apply for API Key of Android map

Open http://code.google.com/intl/zh-CN/android/maps-api-signup.html, and then input the MD5 fingerprint obtained in the former step. Finally, get the API Key of Android map: 0_m9JB9jLjWCLqj_MAoPgdLf8D2aM9gPjG8Wuuw.

(3) Create a primary key of View to display map 
<view android:id="@+id/mv"

class="com. google. android. maps. Map View"

android:layout_width="fill_parent"

android:layout_height="fill_parent"

android:layout_weight="1"

android:apiKey="0_m9JB9jLjWCLqj_MAoPgdLf8D2aM9gPjG8Wuuw"/>

\subsection{The Implementation of Stadium-based Optimal Travel Route}

This function is the core of the system. Corresponding to the design of spatial accessibility algorithm in the previous chapter, algorithm based on Eclipse environment, ArcGIS Server platform will be implemented in the way of J2EE in this chapter. Shortest Path Analysis belongs to category of ArcGIS Network Analysis. ArcGIS network analysis is divided into two categories: geometric-based network analysis and network datasets network analysis. According to the published map services, network analysis can only be realized on the premise that traffic road network model is established in Shijiazhuang. Network analysis is characterized by the analysis of the shortest path.

For example, a user intending to watch the badminton event needs to go to Stadium of Hebei University of Science and Technology, Stadium of Railway Institute and Stadium of Hebei Medical University in turn. The ranges of iterative buffer search are in the order of 100 meters, 200 meters, 400 meters, 800 meters. The three stadiums find their bus stops around them after respectively experiencing iterative buffer search for four times, two times, three times, as shown in Figure 9.

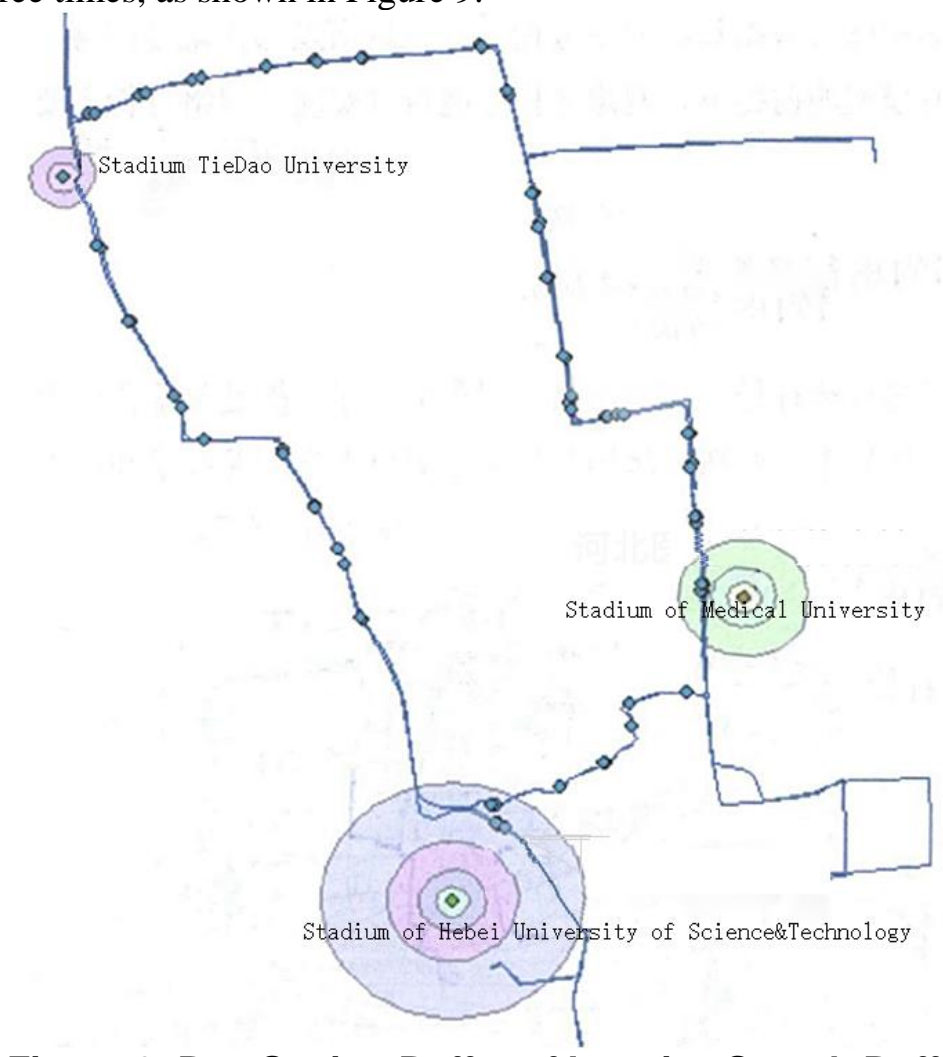

Figure 9. Bus Station Buffer of Iterative Search Buffer

Then supposing that the user is in the campus of Hebei University of Science and technology, the order chosen by the user is as follows: stadium of Hebei University of Science and technology, stadium of Railway Institute and Stadium of Hebei Medical University. The path planning function is implemented by invoking the optimal travel route design algorithm devised in the former part, as shown in figure 10. 


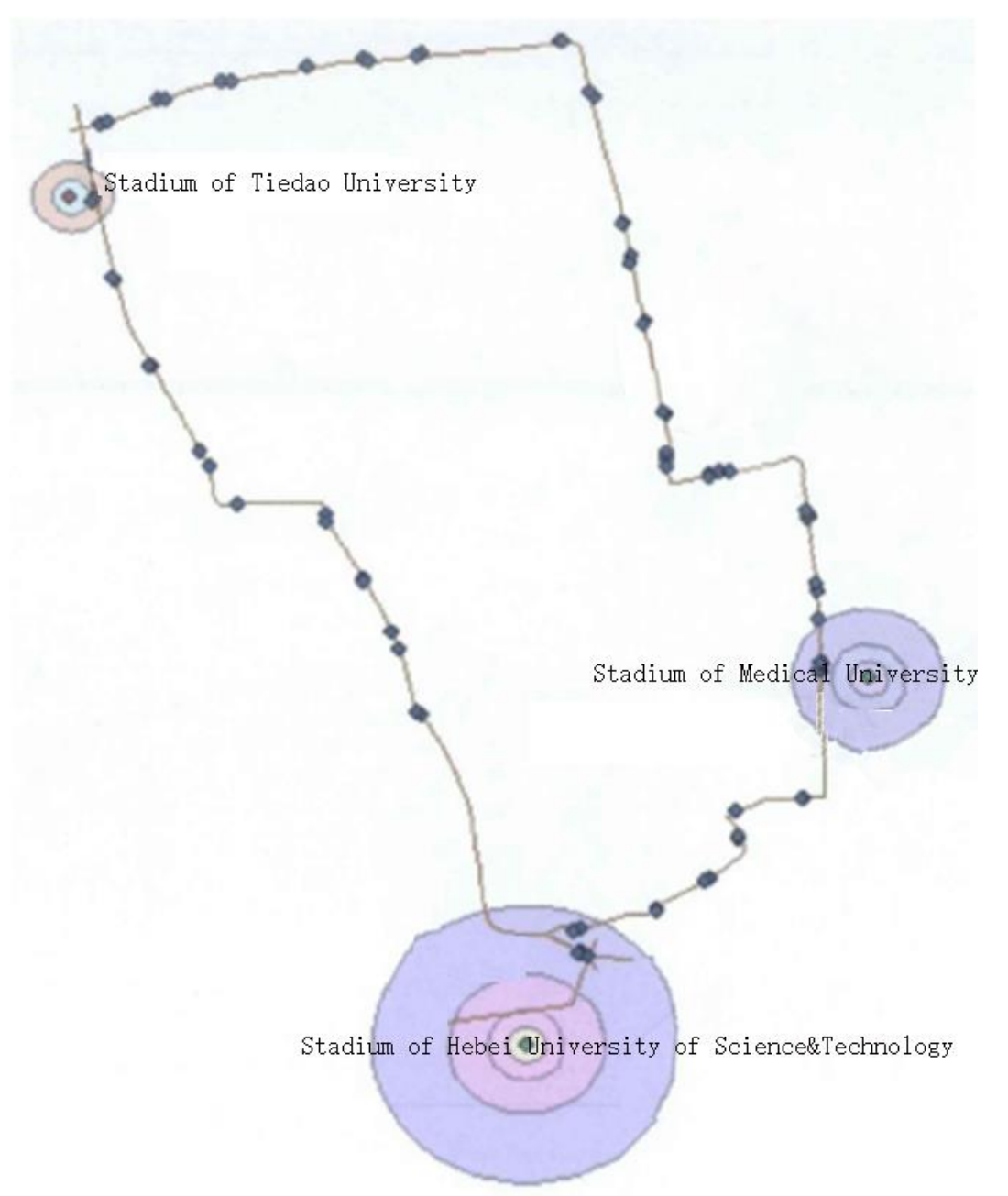

Figure 10. The Realization of the Best Travel Routes

\section{Conclusion}

This paper aims to provide stadium information inquiry service for users. Based on the basic geographic data, traffic thematic data and stadiums data in Shijiazhuang and combined with WebGIS and mobile GIS technology, a city stadium intelligent guide service system is developed. This paper also researches on the buffer analysis algorithm, bus transfer algorithm based on breadth-first and Dijkstra shortest path algorithm. Then according to the spatial distribution characteristics of stadiums in Shijiazhuang, it devises the optimal travel routes design algorithm based on stadiums. This algorithm promotes the implementation of the WebGIS-based stadiums service system. The path planning module incorporates bus transfer algorithm based on breadth-first and Dijkstra shortest path algorithm based on marked nodes. Then it works out an optimal travel route according to the order of destinations chosen by users, which provides convenience for users' travel. The mobile GIS WebGIS technology is embedded into the designed system, which facilitates the system to make full use of the advantages of mobile Internet and GPS technology. This embedded technology also helps to perfect the query and analysis functions of the system and makes it possible to provide real-time and twenty-four-hour service for users. In a word, this system lays a foundation for the construction of smart city. 


\section{References}

[1] P. C. McIntosh, "Landmarks in the history of physical education", Routledge, (2013).

[2] N. M. Toure, A. Kane and J. F. Noel, "Water-poverty relationships in the coastal town of Mbour (Senegal): Relevance of GIS for decision support", International Journal of Applied Earth Observation and Geoinformation, vol. 14, (2012), pp. 33-39.

[3] Z.-X. Fang, X.-L. Zong and Q.-Q. Li, "Hierarchical multi-objective evacuation routing in stadium using ant colony optimization approach", Journal of Transport Geography vol. 19, (2011), pp. 443-451.

[4] I. A. Yeo, S. H. Yoon and J. J. Yee, "Development of an Environment and energy Geographical Information System (E-GIS) construction model to support environmentally friendly urban planning", Applied Energy, vol. 104, (2013), pp. 723-739.

[5] J. C. García-Palomares, J. Gutiérrez and C. Mínguez, "Identification of tourist hot spots based on social networks: A comparative analysis of European metropolises using photo-sharing services and GIS", Applied Geography, vol. 63, (2015), pp. 408-417.

[6] M. T. Françoso, D. C. Costa and M. M. Valin, "Use of Open Source Software for the Development of Web GIS for Accessibility to Tourist Attractions", Journal of Civil Engineering and Architecture, vol. 7, no. 4, (2013), p. 472.

[7] R. K. Das, M. R. Patra and M. Routray, "Integrating WebGIS with service oriented rural information grid: experience sharing", Proceedings of the 4th International Conference on Theory and Practice of Electronic Governance, ACM, (2010).

[8] J. M. Wairiuko, “Application Of Web-GIS In Mapping Older Persons Cash Transfer (OPCT) Case Study: Langata Constituency, Kenya, University of Nairobi, (2013).

[9] Z. Chen, J. Wang and Z. Liu, "Multi-Index Irrigation Information Management System Based on WebGIS”, Scientia Agricultura Sinica, vol. 9, no. 4, (2013).

[10] K. Stereńczak, R. Zapłata and M. Sztampke, "Laser Discoverers"-Web - based User - generated Content in Heritage Detection in Poland", Transactions in GIS, (2016).

[11] P. Jankowski, M. Czepkiewicz and M. Młodkowski, "Geo - questionnaire: A Method and Tool for Public Preference Elicitation in Land Use Planning", Transactions in GIS, (2015).

[12] B. B. Walker, N. Schuurman and S. M. Hameed, "A GIS-based spatiotemporal analysis of violent trauma hotspots in Vancouver, Canada: identification, contextualisation and intervention", BMJ open, vol. 4, no. 2, (2014).

[13] L. Yin, "Integrating 3D visualization and GIS in planning education", Journal of Geography in Higher Education, vol. 34, no. 3, (2010), pp. 419-438.

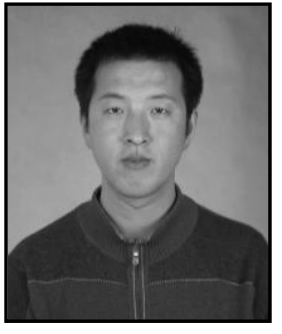

Guofeng WU. He is a lecturer of Shijiazhuang Tiedao University, and his main research direction is sports training and teaching. His contact address is Shijiazhuang Tiedao University, Shijiazhuang, Hebei, China, 050081.

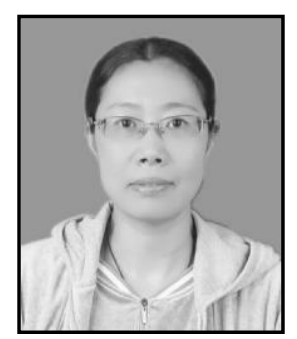

Zhishuang LI. She is an associate professor of Shijiazhuang Tiedao University, and her main research direction is sports training and teaching. Her contact address is Shijiazhuang Tiedao University, Shijiazhuang, Hebei, China, 050081. 